\title{
Le Big Five Inventory français permet-il d'évaluer des facettes en plus des cinq grands facteurs?
}

\author{
Robert Courtois ${ }^{12}$, Jean-Michel Petot ${ }^{3}$, Baptiste Lignier ${ }^{4}$, Gilles Lecocq $^{56}$, Odile Plaisant ${ }^{78}$
}

${ }^{1}$ Université François Rabelais de Tours, PRES Centre-Val de Loire Université, Département de psychologie, EA 2114 'Psychologie des âges de la vie', F-37041 Tours cedex 1, France

${ }^{2} \mathrm{CHRU}$ de Tours, Clinique Psychiatrique Universitaire, F-37044 Tours cedex 9, France

${ }^{3}$ Université de Paris-Nanterre, Laboratoire de Psychologie clinique et Psychopathologie (EA 4430), F92001 Nanterre Cedex, France

${ }^{4}$ Laboratoire Psychopathologie et Psychologie Médicale, LPPM, EA 4452, Univ. Bourgogne FrancheComté, F-21000, Dijon, France

${ }^{5}$ ILEPS-Cergy (L'Ecole Supérieure des Métiers du Sport), F-95000 Cergy, France

${ }^{6}$ Université de Picardie Jules Verne, CRP-CPO EA 7273 'Cognition, Psychisme et Organisations', F80025 Amiens cedex 1, France

${ }^{7}$ Université Paris-Descartes, Sorbonne Paris-Cité, ANCRE, EA 4465, URDIA, F-75006 Paris, France

8 Groupe hospitalier Pitié-Salpêtrière, Assistance publique-Hôpitaux de Paris, Consultation d'évaluation et de traitement de la douleur, unité d'épileptologie, département de psychiatrie adulte, F-75013 Paris, France

Correspondance à :

Robert Courtois

Université François Rabelais

Département de Psychologie, EA 2114,

3, rue des tanneurs - BP 4103, F-37041 Tours Cedex 1

Email : robert.courtois@univ-tours.fr

Nombre de mots : 6430 (3922 sans le résumé, l'abstract, les références, tableaux et figure) 


\section{Le Big Five Inventory français permet-il d'évaluer des facettes en plus des cinq grands facteurs?}

\section{Does the French Big Five Inventory evaluate facets other than the Big Five factors?}

\section{Résumé}

Introduction. Le Big Five Inventory (BFI) de John (1991) représente un des outils les plus consensuels de l'évaluation dimensionnelle de la personnalité. Les cinq grandes dimensions qu'il mesure sont: l'Extraversion, l'Agréabilité, le Caractère consciencieux, le Névrosisme et l'Ouverture. Soto et John (2009) ont montré qu'il était possible de considérer deux facettes par dimension du BFI. L'objectif de cette étude est de savoir s'il est possible de retrouver ces dix facettes dans un échantillon français, en s'appuyant sur les corrélations avec les facettes équivalentes du NEO PI-R (NEO Personality Inventory Revised).

Méthode. Le premier échantillon constitué de 360 étudiants de l'ILEPS (Institut Libre d'Education Physique Supérieure) et de I'Université de Tours (en psychologie) a permis de tester la validité externe des facettes du BFI-Fr en lien avec celles du NEO PI-R. Le second échantillon constitué de 142 étudiants de psychologie de l'Université de Tours a permis de rechercher la validité temporelle (test et retest). Le troisième échantillon constitué de 252 étudiants en psychologie de l'Université de ParisNanterre a permis de tester la forme hétéro-évaluative du BFI-Fr.

Résultats et discussion. Le BFI-Fr permet d'évaluer neuf des dix facettes proposées par Soto et John : Activité et Assertivité pour la dimension Extraversion; Altruisme et Compliance pour la dimension Agréabilité ; Ordre et Autodiscipline pour la dimension Caractère consciencieux; Anxiété pour la dimension Névrosisme; Ouverture à l'esthétique et Ouverture aux idées pour la dimension Ouverture. L'étude a permis de définir une facette Instabilité émotionnelle en remplacement de la facette Dépression du Névrosisme qui ne présentait pas de qualités psychométriques suffisantes.

\section{Mots-clés}

$\mathrm{BFI}$; Cinq grandes dimensions de personnalité ; Facettes, Aspects ; NEO PI-R

\section{Keywords}

Personality; Five factor model; Facets; Aspects; NEO PI-R 


\section{Abstract}

Introduction. The Big Five Inventory (BFI) developed by John et al. (1991) is one of the most widely accepted tools for assessing dimensions of personality. It comprises 44 items that assess five broad dimensions of personality (the Big Five Factors): Extraversion, Agreeableness, Conscientiousness, Neuroticism and Openness to experience. Based on correlations with the facets described in the NEO Personality Inventory Revised (NEO PI-R), another Big Five assessment tool with 240 items and 6 facets per dimension, Soto and John (2009) showed that the dimensions in the BFI could be divided into two facets each (ten facets altogether). These results are in line with those of DeYoung, Quilty and Peterson (2007), who ran factorial analyses with all the NEO PI-R facets and the International Personality Item Pool (IPIP) and identified ten intermediate factors (between facets and dimensions) which they called "aspects" (two per dimension). The goal of the present study is to investigate the ten facets described by Soto and John in a French sample, using the French version of the BFI (BFI-Fr), which has good psychometric properties, and to check whether the pattern of correlations of these facets with the NEO PI-R match those of the American version.

Method. We created three groups. The first comprised 360 students from the ILEPS (Institut Libre d'Education Physique Supérieure) and Tours University (psychology undergraduates). Participants (mean age 21.1 years \pm 2.30 ; 58\% women) completed the BFI-Fr and the NEO PI-R. The second comprised 142 psychology students from Tours University (mean age 20.6 years $\pm 1.78 ; 81 \%$ women); they completed the BFI-Fr twice, two weeks apart (test and retest). The third comprised 252 psychology students from Paris-Nanterre University (mean age 23 years $\pm 4.2 ; 89 \%$ women) who described a total of 405 people they knew well (mean age $35.2 \pm 10.8 ; 49 \%$ women) using the peerreport format of the BFI-Fr.

Results. In the self-report format, eight of Soto and John's ten aspects had acceptable internal consistency (based on Guildford's (1954) internal consistency criteria, due to the small number of items), with Cronbach's $\alpha$ between .60 and .86 and test-retest correlations between .71 and .89 , showing satisfactory temporal stability. We found a single facet for Extraversion (Assertiveness), two for Agreeableness (Altruism and Compliance), two for Conscientiousness (Self-Discipline and Order), one for Neuroticism (Anxiety), and two for Openness to Experience (Openness to aesthetics and Openness to ideas). Based on their convergence with the corresponding facets in the NEO PI-R, these eight facets showed satisfactory external validity. With regard to the peer-report format, the Activity facet of Extraversion, which did not have sufficient internal consistency in the self-report format, had acceptable properties (i.e. 9 out of 10 facets). Only the Depression facet of Neuroticism still had insufficient internal consistency. In this study, we proposed an improvement of two facets (Activity and Compliance) and added one facet specific to the French version (Emotional Instability) in place of the Depression facet.

Discussion. We showed that the BFI-Fr can be used to assess nine of the ten facets described by Soto and John. We also identified an Emotional Instability facet, replacing the Depression facet of Neuroticism. DeYoung et al. (2007) considered that anxiety and depression are indissociable and can be represented by a Neuroticism aspect they labeled Withdrawal. They suggested a second aspect of this dimension they called Volatility (with the N2 Angry Hostility facet of the NEO PI-R as main marker and the N5 Impulsiveness and N3 Depression as secondary markers). The Emotional Instability facet we found corresponds closely to the N2 Angry Hostility facet of the NEO PI-R and appears to be a satisfactory marker of DeYoung et al.'s (2007) Volatility aspect. Although this study has limitations, particularly related to the samples (students), the BFI-Fr facets (derived from those defined by Soto and John in the BFI or proposed as improvements on the original facets) match the corresponding NEO PI-R facets and can also be seen as main markers of the aspects defined by DeYoung et al. 


\section{Introduction}

Au cours des 25 dernières années, le modèle de personnalité dit des cinq grands facteurs (Big ve Factors) s'est progressivement imposé [1-5]. Ce modèle considère que tous les traits de personnalité antérieurement décrits se rattachent à l'un des grands domaines suivants: Extraversion, Agréabilité, Conscienciosité ou Caractère consciencieux, Névrosisme ou Instabilité émotionnelle et Ouverture. Le nom de ces domaines et ce qu'ils recouvrent peut varier légèrement en fonction des outils auquel ils se réfèrent et de leur origine [6]. Chacun d'eux pouvant être décrit au moyen d'un nombre relativement faible d'items portant sur leurs aspects les plus caractéristiques, il est possible d'évaluer la structure globale de la personnalité au moyen d'inventaires courts, comme le Big Five Inventory (BFI) de John [7] qui ne comporte que 44 items et qui, traduit dans de nombreuses langues, est l'un des inventaires de personnalité les plus utilisés dans le monde. Plaisant et al. [8] en ont validé la version française en 45 items, le BFI-Fr.

Mais ces grands domaines ont un degré élevé de généralité et d'abstraction. Chacun regroupe des traits relativement hétérogènes qui avaient souvent été considérés comme des facteurs ultimes dans les classifications antérieures [9-11]. L'affirmation du caractère exhaustif d'un modèle en cinq facteurs très généraux débouche donc logiquement sur une représentation hiérarchique des traits de personnalité, dans laquelle les traits de niveau supérieur subsument plusieurs traits plus restreints et plus proches des réalités observables [12,13]. Ainsi, Costa et McCrae ont conçu le questionnaire de personnalité NEO PI-R (NEO Personality Inventory Revised) [14] de manière à mesurer à la fois les cinq grands domaines et un ensemble de 30 traits de niveau inférieur qu'ils ont nommés des facettes. L'avantage de tels instruments est de donner une représentation quasiment exhaustive de la personnalité, mais cela nécessite un grand nombre d'items (240 pour le NEO PI-R) et donc un temps d'administration élevé.

Plus récemment, Soto et John [15] ont avancé qu'il était possible de regrouper, à l'intérieur de chacune des échelles du BFI, certains items évaluant plus particulièrement un 
type d'attitudes ou de conduites pouvant correspondre à l'un de ces facteurs de niveau inférieur et ont examiné la correspondance avec les facettes du NEO PI-R de dix de ces regroupements d'items. La possibilité d'évaluer, avec seulement 44 items, non seulement les cinq grands domaines mais également dix facettes, augmente considérablement l'intérêt du BFI pour l'évaluation clinique et pour la recherche.

Par ailleurs, le fait que ces dix facettes se répartissent entre les cinq grands domaines, à raison de deux facettes par domaine, doit être rapproché des résultats de DeYoung, Quilty et Peterson [16] qui, ayant conduit des analyses factorielles sur l'ensemble des 75 facettes du NEO PI-R et de la liste internationale d'items de personnalité (IPIP : International Personality Items Pool) [17], affirment l'existence de facteurs d'un niveau intermédiaire entre les cinq grands domaines et les facettes. II y aurait deux de ces facteurs dans chaque domaine. Afin de clarifier l'exposé, nous ferons suivre désormais le nom des facettes proposées par Soto et John de l'indication « du BFI », le terme « aspect » renverra systématiquement aux facteurs de niveau intermédiaire postulés par de DeYoung et ses collègues, et les noms des facettes du NEO PI-R seront précédées du code indiquant leur domaine d'appartenance ( $\mathrm{N}$ pour Névrosisme-Instabilité émotionnelle, E pour Extraversion, O pour Ouverture, A pour Agréabilité, C pour Conscienciosité) et leur numéro dans le domaine considéré.

Les deux approches sont convergentes en ce qui concerne l'Agréabilité, la Conscienciosité et l'Ouverture : l'Altruisme du BFI correspond à l'aspect Compassion, dont le marqueur le plus important est précisément A3 Altruisme ; la Compliance du BFI correspond à l'aspect Politesse, dont le marqueur principal est la facette $A 4$ Compliance (voir tableau 1). La facette Ordre du BFI correspond à l'aspect homonyme (Orderliness) dont le marqueur principal est la facette C2 Ordre. L'Autodiscipline du BFI correspond à l'aspect Efficacité (Industriousness) dont le marqueur principal est C5 Autodiscipline. L'Ouverture à l'Esthétique du BFI correspond à l'aspect Ouverture, ce dernier ayant sa corrélation la plus élevée avec O2 Ouverture à l'esthétique ; la facette Ouverture aux idées du BFI correspond à l'aspect 
Intellect, qui a sa plus forte corrélation avec la facette O5 Ouverture aux idées. II n'y a pas de coïncidence, en revanche, dans les domaines de l'Extraversion et du Névrosisme. Soto et John distinguent l'Assertivité et l'Activité, alors que DeYoung et ses collègues opposent l'Assertivité à l'Enthousiasme, et considèrent que l'Activité est une des composantes de l'aspect Assertivité. Dans le domaine Névrosisme, Soto et John proposent des facettes permettant de mesurer séparément l'Anxiété et la Dépression. DeYoung et ses collègues considèrent que ces deux facettes relèvent du même aspect, qu'ils nomment Retrait, l'autre aspect du névrosisme étant l'Instabilité (Volatility) qui a des corrélations élevées avec N2 Colère-Hostilité et N5 Impulsivité. Les deux approches sont donc différentes : Soto et John n'ont pas cherché à évaluer des réalités intermédiaires entre les facettes et les domaines comme le sont les aspects de DeYoung et de ses collègues. Cependant, dans trois des domaines, les deux facettes du BFI relèvent d'aspects différents et pourraient d'une certaine manière permettre une évaluation de ces aspects à partir de leur facette la plus caractéristique.

Insérer ici environ le tableau 1

L'objectif de cette étude est d'examiner si le BFI-Fr permet l'évaluation de facettes. II s'agit d'abord d'établir s'il est possible de retrouver dans un échantillon français les dix facettes établies par Soto et John à partir de leur échantillon américain et si le «pattern » des corrélations de ces facettes avec celles du NEO PI-R correspond bien à celui de la version américaine. II s'agit ensuite de chercher si certains regroupements d'items permettent de définir des facettes qui seraient propres au BFI-Fr.

\section{Méthode}

\section{Participants et procédure}

L'échantillon 1 est constitué de 360 étudiants de l'ILEPS (Institut Libre d'Education Physique Supérieure ou Ecole Supérieure des Métiers du Sport) et de l'Université de Tours 
(en psychologie), âge moyen de 21,1 ans $\pm 2,30$ (étendue de 18,3 à 45,5 ) avec $58 \%$ de

femmes et $81 \%$ de sujets en Licence. Ils ont rempli conjointement le BFI-Fr [8] (forme autoévaluée) et le NEO PI-R [14]. L'échantillon 2 est constitué de 142 étudiants en Licence de psychologie de l'Université de Tours, avec un âge moyen de 20,6 \pm 1,78 (étendue de 18,3 à 34,4 et $81 \%$ de femmes) qui ont rempli le BFI-Fr (forme auto-évaluée) à deux reprises, à deux semaines d'intervalle. Enfin l'échantillon 3 est constitué par des données d'hétéroévaluation : 252 étudiants en psychologie de l'université de Paris Nanterre, avec un âge moyen de 23 ans $\pm 4,2$ (étendue de 19 à 46 ans, dont $89 \%$ de femmes), ont décrit 405 personnes de leur entourage proche au moyen de la forme hétéro-évaluative du BFI-Fr [18]. Les personnes décrites (dont 199 femmes) ont un âge moyen de 35,2 \pm 10,8 (étendue de 19 à 65 ans). Quarante-deux de ces personnes ont été décrites une deuxième fois après un délai de trois semaines. L'échantillon 1 permettra l'étude de la validité convergente avec le NEO PI-R, l'échantillon 2 l'étude des corrélations test-retest des facettes investiguées, l'échantillon 3 d'évaluer une démarche similaire de recherche de facettes à partir de la version hétéro-évaluative du BFI-Fr.

\section{Instruments}

Le BFI-Fr est la version française en 45 items [8] du Big Five Inventory (BFI) de John [7]. Les réponses sont cotées selon une échelle de Likert à cinq niveaux, allant de $1=$ désapprouve fortement à 5 = approuve fortement. Les scores des échelles sont la moyenne des scores aux items constituant ces échelles, après recodage en sens inverse des items qui visent le pole négatif du facteur (par exemple « cherche des histoires aux autres » pour l'Agréabilité). Les propriétés psychométriques du BFI-Fr sont excellentes avec des coefficients de cohérence interne variant de 0,77 à 0,83 et des corrélations avec les grands domaines du NEO PI-R variant de 0,69 à 0,79. La forme hétéro-évaluative du BFI-Fr a été validée récemment et ses cinq échelles présentent une cohérence interne équivalente à celle de la version auto-évaluative et une fidélité test-retest satisfaisante [18]. 
Le NEO PI-R [14], adapté dans de nombreux pays dont la France [19], est un des questionnaires de personnalité les plus utilisés dans le monde. C'est un questionnaire de 240 items qui mesure les cinq domaines de la personnalité ainsi que six facettes de chaque domaine. Les réponses sont cotées sur une échelle de Likert allant de 0 = fort désaccord à 4 $=$ fort accord. Les scores bruts aux échelles sont obtenus en faisant la somme des items constitutifs, après recodage des items inverses.

\section{Analyses statistiques}

Afin d'établir la validité des facettes du BFI-Fr, nous avons estimé la validité interne des regroupements d'items en examinant leur cohérence interne au moyen du coefficient $\alpha$ de Cronbach [20] et leur stabilité temporelle à partir de la fidélité test-retest mesurée par les coefficients de corrélation entre scores obtenus lors de la première et de la deuxième passation de l'instrument. Comme aucune des facettes du BFI ne comporte plus de cinq items et que certaines n'en contiennent que deux, et comme le coefficient $\alpha$ sous-estime la cohérence interne des échelles comportant un faible nombre d'items [21] nous avons retenu le critère d'évaluation de la cohérence interne de Guilford qui fixe celle-ci à 0,60 pour les études exploratoires [22-24].

Pour la fidélité test-retest, nous avons retenu le critère usuel $r \geq 0,70$. Nous avons évalué la validité externe des facettes de la forme auto-évaluative du BFI-Fr au moyen de la corrélation entre les scores aux facettes du BFI et les scores aux facettes correspondantes, généralement homonymes, du NEO PI-R.

\section{Résultats}

\section{Forme auto-évaluative}


Dans la forme auto-évaluative, comme le montre le tableau 2, huit des dix facettes de

Soto et John présentent des qualités psychométriques internes acceptables (si on retient le critère d'évaluation de la cohérence interne de Guilford), avec des a compris entre 0,60 et 0,86 et des corrélations test-retest comprises entre 0,71 et 0,89 indiquant des stabilités temporelles satisfaisantes. On retrouve une seule facette pour l'Extraversion (Assertivité), deux pour l'Agréabilité (Altruisme et Compliance), deux pour la Conscienciosité ou Caractère consciencieux (Ordre et Autodiscipline), une seule pour le Névrosisme (Anxiété) et deux pour l'Ouverture (Ouverture à l'esthétique et Ouverture aux idées) (voir figure 1). Les facettes Activité pour l'Extraversion et Dépression pour le Névrosisme ne présentent pas de cohérence ou consistance interne suffisante. On notera que la cohérence de chaque domaine est bonne : respectivement de 0,$84 ; 0,78 ; 0,83 ; 0,86 ; 0,82$ pour les domaines $E$, A, C, N, et O, y compris en phase de retest (respectivement : 0,$85 ; 0,78 ; 0,81 ; 0,85 ; 0,86$ ).

Insérer ici environ le tableau 2 et la figure 1

Les qualités psychométriques des facettes Activité et Compliance décrites par Soto et John [15] peuvent être améliorées par l'addition d'un item. L'ajout de l'item 36 (sociable, extraverti) pour la première qui est constituée des items 11 (plein d'énergie) et 16 (communique beaucoup d'enthousiasme) améliore la cohérence interne. Nous l'avons dénommée dans le tableau 2 " Enthousiasme-Fr ». De même, l'ajout de l'item 27 (parfois dédaigneux, méprisant) à la facette Compliance permet d'obtenir un a acceptable et une corrélation test-retest satisfaisante pour une facette « Compliance-Fr ». Enfin, la proximité du contenu et la corrélation entre deux items suggèrent la possibilité de définir une facette qui serait propre à la version française : Instabilité émotionnelle-Fr composée des items 24 (tempéré, pas facilement troublé) et 29 (lunatique, d'humeur changeante). Cette facette présente une cohérence interne et une stabilité temporelle acceptables. Elle peut représenter une alternative à la facette Dépression et un marqueur de l'aspect Volatilité.

\section{Forme hétéro-évaluative}


Pour la forme hétéro-évaluative, les propriétés psychométriques internes de neuf des dix facettes proposées par Soto et John [15] sont satisfaisantes, avec des a compris entre 0,71 et 0,84 et des corrélations test-retest comprises entre 0,72 et 0,88 , comme le montre le tableau 2. La facette Dépression présente au contraire une cohérence interne insuffisante.

\section{Validité convergente avec le NEO PI-R}

En ce qui concerne la validité convergente, toutes les facettes de Soto et John ont dans notre échantillon (pour la forme auto-évaluative) leur corrélation la plus élevée avec la facette homonyme du NEO PI-R et cette corrélation est égale ou supérieure à 0,60 pour neuf d'entre elles et supérieure à 0,50 pour la dernière. II en est de même pour les facettes propres à la version française (voir tableau 2). La facette Compliance-Fr présente sa corrélation la plus élevée avec A4 Compliance. La facette Enthousiasme-Fr a ses corrélations les plus élevées avec E1 Chaleur et E6 Emotions positives. L'examen systématique des corrélations entre les items du BFI-Fr et les facettes du NEO PI-R a permis de proposer une facette propre à la version française, Instabilité-Fr fortement corrélée à la facette N2 Colère-Hostilité.

\section{Discussion}

L'objectif de cette étude était d'établir si les facettes du BFI proposées par Soto et John [15] se retrouvent dans la version française de l'inventaire et, secondairement, d'étudier la possibilité de définir des facettes propres au BFI-Fr. Huit des dix facettes originales de Soto et John sont retrouvées avec une consistance interne suffisante et une bonne stabilité temporelle. Nous avons proposé une amélioration de deux facettes et l'ajout d'une facette propre à la version française. La validité externe des huit facettes présentant une cohérence interne suffisante, évaluée sur la base de leur convergence avec les facettes correspondantes du NEO PI-R, est satisfaisante. Toutes ont leur corrélation la plus élevée avec la facette homonyme du NEO PI-R et ces corrélations sont comprises entre 0,58 et 
0,78 , ce qui est très proche des corrélations observées dans la version américaine (entre 0,58 pour la Compliance et 0,72 pour l'Autodiscipline).

On retiendra donc que le BFI-Fr permet d'évaluer neuf des dix facettes proposées par Soto et John: Assertivité et Activité pour l'Extraversion; Altruisme et Compliance pour l'Agréabilité ; Ordre et Autodiscipline pour la Conscienciosité ou Caractère consciencieux ; Anxiété pour le Névrosisme et Ouverture à l'esthétique et Ouverture aux idées pour l'Ouverture. Nous avons également pu définir une facette Instabilité émotionnelle qui est un marqueur satisfaisant de l'aspect Volatilité de DeYoung et al. [16].

La forme hétéro-évaluée du BFI-Fr présente des résultats meilleurs que la forme auto-évaluée même si la facette Dépression présente aussi une cohérence interne insatisfaisante. Nous faisons l'hypothèse que ces meilleurs résultats pourraient s'expliquer par le fait que les sujets qui décrivent des proches pourraient avoir tendance à «forcer » légèrement certains traits et que l'idée qu'ils se font de la personne demeure assez constante (davantage que celle que des personnes peuvent avoir d'elles-mêmes).

Pour ce qui concerne l'Extraversion, Soto et John ont proposé de distinguer deux facettes: l'Assertivité et l'Activité. La première présente des qualités psychométriques internes satisfaisantes et une corrélation très élevée avec la facette homonyme du NEO PI$\mathrm{R}$, dont elle est par conséquent un marqueur satisfaisant. La seconde, en revanche, présente des qualités psychométriques moins bonnes et une corrélation plus faible avec la facette homonyme du NEO PI-R. L'ajout d'un item permet d'améliorer ses qualités psychométriques et ses corrélations avec les facettes E1 Chaleur, E6 Emotions positives, qui font partie de l'aspect Enthousiasme. Ce pourrait être un choix permettant de disposer ainsi en version française d'une seconde facette différente (pour partie) de la version américaine.

En ce qui concerne l'Agréabilité, la facette Altruisme présente une cohérence interne et une stabilité temporelle satisfaisantes, une corrélation élevée avec A3 Altruisme et avec A6 Sensibilité qui sont également les deux premiers marqueurs de l'aspect Compassion. Elle 
permet donc une mesure satisfaisante de l'Altruisme au niveau des facettes et de la interne insuffisante, mais après ajout d'un item, la facette Compliance-Fr présente des propriétés psychométriques acceptables et un pattern de corrélations qui diffère légèrement de celui de l'aspect homonyme, mais qui correspond bien au concept de compliance, la corrélation la plus élevée étant avec A4 Compliance, suivie par une corrélation négative avec N2 Colère-Hostilité.

La première facette du Caractère consciencieux, Ordre, présente une cohérence acceptable. L'un des deux items dont elle est constituée, l'item 18 (tend à être désorganisé) a une corrélation si élevée $(0,76)$ avec la facette C2 Ordre qu'il serait possible d'évaluer à partir de ce seul item, non seulement cette facette, mais aussi l'aspect Ordre dont le pattern de corrélations est exactement identique : d'abord C2 Ordre, puis C5 Autodiscipline et C3 Sens du devoir. La deuxième facette originale, Autodiscipline, a des propriétés psychométriques acceptables et sa validité est confirmée par sa corrélation la plus élevée avec C5 Autodiscipline et un pattern de corrélations proche de l'aspect homonyme.

Si dans le domaine Névrosisme, Soto et John ont défini deux facettes évaluant respectivement l'Anxiété et la Dépression et que la facette Anxiété présente une cohérence interne et une stabilité temporelle très satisfaisantes dans la version française - avec une corrélation très élevée avec N1 Anxiété, mais aussi avec l'aspect Retrait, dont le marqueur principal est N3 Dépression, suivi par N6 Vulnérabilité au stress et N1 Anxiété, la facette Dépression en revanche présente une cohérence interne insuffisante. II est clair que la facette Anxiété ne correspond pas seulement à N1 Anxiété, mais qu'elle est en réalité un marqueur de l'ensemble anxiété-dépression que DeYoung et ses collègues considèrent comme formant l'aspect Retrait du Névrosisme. Cela est cohérent avec plusieurs recherches qui ont souligné, notamment sous l'impulsion de Watson et de Clark [25-28], l'extrême difficulté de différencier l'anxiété et la dépression, à la fois sur le plan théorique et sur le plan de la pratique évaluative. Au Retrait, DeYoung et ses collègues opposent l'Instabilité dont le 
marqueur principal est N2 Colère-Hostilité et les marqueurs secondaires N5 Impulsivité et N3 interne et la stabilité temporelle sont acceptables, présente un pattern de corrélations qui en fait une bonne candidate pour l'évaluation de la facette N2 Colère-Hostilité comme de l'aspect Instabilité.

Dans le domaine d'Ouverture, les deux facettes originales, Esthétique et Idées, présentent des qualités psychométriques internes acceptables. Elles ont leur corrélation la plus élevée avec la facette homonyme du NEO PI-R et leurs patterns de corrélations avec les facettes du NEO PI-R correspondent très exactement à ceux des deux aspects Ouverture et Intellect. Elles permettent donc l'évaluation non seulement des facettes 02 Esthétique et 05 Idées, mais aussi de ces aspects.

Comment expliquer les trois différences que nous observons entre les facettes du BFI américain original et celles que nous retrouvons dans le BFI français ? L'échantillon initial du BFI américain était également constitué d'étudiants. La principale de ces différences concerne la facette Dépression que nous ne retrouvons ni dans la version auto-évaluée ni dans la forme hétéro-évaluée du BFI-Fr. Cette facette ne comporte que deux items (items 4 et 29), ce qui peut expliquer en partie le fait qu'elle ait un coefficient de cohérence interne insuffisant. La traduction de l'item 4 ne pose pas de problème (is depressed, blue / est déprimé, cafardeux). Mais l'item 29 (can be moody / peut-être lunatique, d'humeur changeante) a plus de rapport en français avec l'Agréabilité qu'avec le Névrosisme. Sa saturation dans la première dimension est légèrement supérieure (en valeur absolue) à celle dans la seconde. Nous supposons que cela s'explique par le fait que le mot « lunatique » est en partie un faux-ami. En anglais, « moody » évoque clairement la maladie mentale ou une excentricité marquée et ne comporte pas de connotation interpersonnelle [29]. En français, à la notion d'humeur changeante s'ajoute une notion interpersonnelle (versatilité déconcertante pour autrui - dans le sens de mal luné, imprévisible) [30]. 
Il est possible qu'une difficulté de traduction explique la faible cohérence interne de la facette originale américaine Activité dans l'échantillon français qui a rempli la version autoévaluée (la cohérence interne est satisfaisante dans l'échantillon qui a rempli la forme hétéro-évaluative). Cette facette est composée des items 11 (is full of energy / est plein d'énergie) et 16 (generates a lot of enthusiasm / communique beaucoup d'enthousiasme). La traduction du second item, qu'il était impossible de traduire mot-à-mot en français, renforce la connotation interpersonnelle. L'item 36 dont l'ajout à la variante française de cette facette a permis d'obtenir une cohérence interne satisfaisante est un item à contenu interpersonnel (is outgoing, sociable / est sociable, extraverti). De ce fait, la facette change de signification : elle évalue à la fois l'activité et la sociabilité. II n'y a pas lieu de le déplorer, car cela présente un sens et un intérêt théorique puisque les travaux de Goldberg[17] et de DeYoung et al. [16] ont montré qu'à l'intérieur de l'Extraversion, la facette Activité, les facettes «prosociales » (Chaleur, Grégarité) et la facette Emotions positives - dont l'ensemble correspond à l'aspect Enthousiasme de DeYoung et al. [16] - sont beaucoup plus corrélées entre elles qu'elles ne le sont à la facette Assertivité.

II est plus difficile de comprendre pourquoi la facette originale Compliance n'a pas une cohérence interne satisfaisante dans l'échantillon français qui a rempli la forme autoévaluée. II s'agit des items $2 R$ (tends to find fault with others / a tendance à critiquer les autres), 12R (starts quarrels with others / commence facilement à se disputer avec les autres), 17 (has a forgiving nature / est indulgent de nature) pour lesquels nous ne détectons aucun problème de traduction, ni aucune hétérogénéité dans le contenu des items de la version française. L'item qu'il a fallu ajouter pour obtenir une cohérence interne satisfaisante va exactement dans le même sens que les trois précédents. De ce fait, la facette Compliance-Fr a exactement la même signification psychologique que la facette originale américaine. II est possible qu'il existe également des légères différences interculturelles concernant les traits de personnalité, mais nous ne disposons pas d'éléments pour les étayer. 
Cette étude comporte certaines limitations. Tout d'abord, pour évaluer la cohérence

interne des facettes, nous avons adopté le critère de Guilford [22-24], qu'on pourrait considérer comme un peu laxiste. Son emploi nous a semblé justifié dans cette étude par le fait que l'indice a de Cronbach est, de l'avis unanime des experts, partiellement biaisé par le nombre des items et qu'il tend à être d'autant plus bas que le nombre des items est faible [21,24] (ici 2 ou 3 items pour certaines facettes). La stabilité temporelle satisfaisante et des corrélations qui attestent une bonne validité convergente peuvent compenser jusqu'à un certain point une cohérence interne médiocre. Par ailleurs, les participants à cette étude sont tous des étudiants dans les métiers du sport (échantillon 1) ou en psychologie (échantillons 1 à 3), ce qui pourrait autoriser des doutes relatifs à la généralisabilité de nos résultats, et rend donc souhaitable de nouvelles recherches portant sur des échantillons plus représentatifs de la population générale. Enfin, concernant la forme hétéro-évaluée, nous avons souligné que le BFI-Fr étant le seul inventaire de personnalité dont il existe une version en langue française destinée à l'hétéro-évaluation, la recherche de validité convergente des facettes pour la version hétéro-évaluée n’a pas été possible.

En conclusion, on retiendra que les résultats de cette étude suggèrent que le BFI-Fr permet l'évaluation de dix facettes dont neuf sont identiques ou très proches de celles décrites par Soto et John [15]. Non seulement ces facettes correspondent à certaines facettes du NEO PI-R, mais les facettes du NEO PI-R auxquelles elles correspondent sont précisément celles qui sont les marqueurs principaux des aspects définis par DeYoung, Quilty et Peterson [16]. Elles permettent donc une bonne évaluation de ces facteurs de niveau intermédiaire entre les grands domaines et les facettes.

\section{Les auteurs déclarent ne pas avoir de liens d’intérêt.}




\section{Références}

1. Goldberg LR. An alternative "description of personality": The Big-Five factor structure. $J$ Pers Soc Psychol 1990; 59: 1216-1229.

2. Digman JM. Personality Structure: Emergence of the Five-Factor Model. Annu Rev Psychol 1990; 41: 417-440.

3. McCrae RR and John OP. An Introduction to the Five-Factor Model and Its Applications. J Pers 1992; 60: 175-215.

4. John OP, Naumann LP and Soto CJ. Paradigm shift to the integrative big-five trait taxonomy: history, measurement, and conceptual issues. In: John OP, Robins RW and Pervin LA (eds) Handbook of personality: Theory and research. 3 ed. New York, NY, US: Guilford Press, 2008, pp.114-158.

5. Petot J-M. L'intérêt clinique du modèle de personnalité en cinq facteurs. Eur Rev Appl Psychol 1994; 44: 57-63.

6. Plaisant O, Guertault J, Courtois R, et al. Histoire des « Big Five »: OCEAN des cinq grands facteurs de la personnalité ; Introduction du Big Five Inventory Français ou BFIFr. Ann Med Psychol 2010; 168: 481-486.

7. John OP, Donahue EM and Kentle RL. The Big Five Inventory--Versions 4a and 54. 1991. Berkeley, CA, US: University of California, Institute of Personality and Social Research.

8. Plaisant $\mathrm{O}$, Courtois $\mathrm{R}$, Réveillère $\mathrm{C}$, et al. Validation par analyse factorielle du Big Five Inventory français (BFI-Fr). Analyse convergente avec le NEO-PI-R. Ann Med Psychol 2010; 168: 97-106.

9. Costa Jr PT and McCrae RR. From catalog to classification: Murray's needs and the fivefactor model. J Pers Soc Psychol 1988; 55: 258-265.

10. McCrae RR and Costa PT. The structure of interpersonal traits: Wiggins's circumplex and the five-factor model. J Pers Soc Psychol 1989; 56: 586-595.

11. McCrae RR and Costa PT. Reinterpreting the Myers-Briggs Type Indicator From the Perspective of the Five-Factor Model of Personality. J Pers 1989; 57: 17-40.

12. Paunonen SV and Ashton MC. Big Five factors and facets and the prediction of behavior. $J$ Pers Soc Psychol 2001; 81: 524-539.

13. Jourdy $R$ and Petot J-M. Relations entre traits de personnalité et dépression au niveau des cinq grands facteurs et de leurs facettes. Evol Psychiatr (Paris) Sous presse. DOI: 10.1016/j.evopsy.2016.01.003.

14. Costa PT and McCrae RR. NEO PI-R professional manual. Odessa, FL, US: Psychological Assessment Resources, Inc, 1992. 
15. Soto CJ and John OP. Ten facet scales for the Big Five Inventory: Convergence with NEO PI-R facets, self-peer agreement, and discriminant validity. J Res Pers 2009; 43: 84-90.

16. DeYoung CG, Quilty LC and Peterson JB. Between facets and domains: 10 aspects of the Big Five. J Pers Soc Psychol 2007; 93: 880-896.

17. Goldberg LR, Johnson JA, Eber HW, et al. The international personality item pool and the future of public-domain personality measures. J Res Pers 2006; 40: 84-96.

18. Lignier B, Petot J-M, Plaisant O, et al. Validation du Big Five Inventory français (BFI-Fr) dans un contexte d'hétéro-évaluation. Ann Med Psychol 2016; 174: 436-441.

19. Costa P, McCrae R and Rolland J. NEO-PI-R. Inventaire de Personnalité révisé. Adaptation Française. Paris, FR: Editions du Centre de Psychologie Appliquée, 1998.

20. Cronbach LJ. Coefficient alpha and the internal structure of tests. Psychometrika 1951; 16: 297-334. journal article. DOI: 10.1007/bf02310555.

21. Cortina J. What is coefficient alpha? An examination of theory and applications. $J$ Appl Psychol 1993; 78: 98-104.

22. Guilford JP. Psychometric methods, 2nd ed. New York, NY, US: McGraw-Hill, 1954, p.ix, 597.

23. Murphy KR and Davidshofer CO. Psychological testing: principles and applications. 5 ed. Upper Saddle River, NJ, US: Prentice Hall, 2001.

24. George D and Mallery M. Using SPSS for Windows step by step: a simple guide and reference 11.0 update. Boston, MA, US: Allyn \& Bacon, 2003.

25. Clark LA and Watson D. Tripartite model of anxiety and depression: Psychometric evidence and taxonomic implications. J Abnorm Psychol 1991; 100: 316-336.

26. Watson D, Clark LA, Weber K, et al. Testing a tripartite model: II. Exploring the symptom structure of anxiety and depression in student, adult, and patient samples. J Abnorm Psychol 1995; 104: 15-25.

27. Watson D and Naragon-Gainey K. Personality, Emotions, and the Emotional Disorders. Clin Psychol Sci 2014; 2: 422-442.

28. Watson D, Weber K, Assenheimer JS, et al. Testing a tripartite model: I. Evaluating the convergent and discriminant validity of anxiety and depression symptom scales. $J$ Abnorm Psychol 1995; 104: 3-14.

29. Pearsall J. The new Oxford dictionary of English. Oxford, UK: Clarendon Press, 1998.

30. Robert P. Dictionnaire alphabétique et analogique de la langue française. Paris, FR: Société du nouveau Littré, 1978. 
Tableau 1. Correspondance entre les facettes du BFI selon Soto et John [15] et les aspects des Big Five selon DeYoung, Quilty et Peterson [16]. Relations avec les facettes du NEO PI-R

\begin{tabular}{|c|c|c|c|}
\hline Domaine & $\begin{array}{l}\text { Facettes du BFI } \\
\text { (Soto et John) }\end{array}$ & $\begin{array}{l}\text { Aspects (DeYoung, } \\
\text { Quilty et Peterson) }\end{array}$ & $\begin{array}{l}\text { Corrélations BFI/ NEO PI-R (données } \\
\text { de Soto et John) }\end{array}$ \\
\hline \multirow{7}{*}{ Extraversion } & \multirow{3}{*}{ Assertivité } & \multirow{3}{*}{ Assertivité } & E3 Assertivité $(0,72)$ \\
\hline & & & E4 Activité $(0,59)$ \\
\hline & & & E6 Emotions positives $(0,43)$ \\
\hline & Activité & $\begin{array}{l}\text { Pas de } \\
\text { correspondance avec } \\
\text { les aspects }\end{array}$ & \\
\hline & \multirow{3}{*}{$\begin{array}{l}\text { Pas de } \\
\text { correspondance } \\
\text { avec le BFI }\end{array}$} & \multirow{3}{*}{ Enthousiasme } & E1 Chaleur $(0,88)$ \\
\hline & & & E2 Grégarité $(0,71)$ \\
\hline & & & E6 Emotions positives $(0,67)$ \\
\hline \multirow{6}{*}{ Agréabilité } & \multirow{3}{*}{ Altruisme } & \multirow{3}{*}{ Compassion } & A3 Altruisme $(0,65)$ \\
\hline & & & A6 Sensibilité $(0,50)$ \\
\hline & & & A1 Confiance $(0,42)$ \\
\hline & \multirow{3}{*}{ Compliance } & \multirow{3}{*}{ Politesse (Politeness) } & A4 Compliance $(0,71)$ \\
\hline & & & A2 Franchise $(0,67)$ \\
\hline & & & A3 Altruisme $(0,64)$ \\
\hline \multirow{6}{*}{$\begin{array}{l}\text { Conscienciosité } \\
\text { ou Caractère } \\
\text { consciencieux }\end{array}$} & \multirow{3}{*}{ Ordre } & \multirow{4}{*}{ Ordre (Orderliness) } & C2 Ordre $(0,79)$ \\
\hline & & & C5 Autodiscipline $(0,55)$ \\
\hline & & & C3 Sens du devoir $(0,50)$ \\
\hline & \multirow{3}{*}{ Autodiscipline } & & C5 Autodiscipline $(0,83)$ \\
\hline & & \multirow{2}{*}{$\begin{array}{l}\text { Efficacité } \\
\text { (Industriousness) }\end{array}$} & C1 Compétence $(0,75)$ \\
\hline & & & C4 Recherche de réussite $(0,65)$ \\
\hline \multirow{7}{*}{ Névrosisme } & \multirow{3}{*}{ Anxiété } & \multirow{3}{*}{ Retrait (Withdrawal) } & N3 Dépression $(0,85)$ \\
\hline & & & N6 Vulnérabilité au stress $(0,78)$ \\
\hline & & & N4 Timidité sociale $(0,76)$ \\
\hline & Dépression & $\begin{array}{l}\text { Pas de } \\
\text { correspondance avec } \\
\text { les aspects }\end{array}$ & \\
\hline & \multirow{3}{*}{$\begin{array}{l}\text { Pas de } \\
\text { correspondance } \\
\text { avec le BFI }\end{array}$} & \multirow{3}{*}{ Instabilité (Volatility) } & N2 Colère-Hostilité $(0,76)$ \\
\hline & & & N5 Impulsivité $(0,59)$ \\
\hline & & & N3 Dépression $(0,57)$ \\
\hline \multirow{6}{*}{ Ouverture } & \multirow{3}{*}{ Esthétique } & \multirow{3}{*}{ Ouverture } & O2 Ouverture à l'esthétique $(0,87)$ \\
\hline & & & O1 Ouverture à l'imagination $(0,64)$ \\
\hline & & & O3 Ouverture aux sentiments $(0,58)$ \\
\hline & \multirow{3}{*}{ Idées } & \multirow{3}{*}{ Intellect } & O5 Ouverture aux idées $(0,76)$ \\
\hline & & & O1 Ouverture à l'imagination $(0,45)$ \\
\hline & & & O4 Ouverture aux actions $(0,36)$ \\
\hline
\end{tabular}


Tableau 2. Propriétés psychométriques des facettes du BFI-Fr (formes auto-évaluée et hétéro-évaluée) et corrélations des facettes auto-évaluées avec les facettes du NEO PI-R

\begin{tabular}{|c|c|c|c|c|c|c|c|}
\hline \multirow[b]{2}{*}{$\begin{array}{l}\text { Facettes du BFI selon Soto } \\
\qquad \& \text { John (2009) }\end{array}$} & \multirow[b]{2}{*}{$\begin{array}{l}\text { Items composant la } \\
\text { facette }\end{array}$} & \multirow[b]{2}{*}{ Dimension } & \multicolumn{3}{|r|}{ Auto-évaluation } & \multicolumn{2}{|c|}{ Hétéro-Evaluation } \\
\hline & & & $\begin{array}{c}\text { a de } \\
\text { Cronbach }\end{array}$ & $\begin{array}{l}r \text { test- } \\
\text { retest }\end{array}$ & $\begin{array}{c}\text { Coefficient de corrélation de la forme } \\
\text { auto-évaluée avec les facettes du } \\
\text { NEO PI-R }\end{array}$ & $\begin{array}{c}\text { a de } \\
\text { Cronbach }\end{array}$ & $\begin{array}{l}r \text { test- } \\
\text { retest }\end{array}$ \\
\hline \multirow{3}{*}{ Assertivité } & & & & & E3 Affirmation de soi $(0,68)$ & & \\
\hline & $1,6 \mathrm{R}, 21 \mathrm{R}, 26,31 \mathrm{R}$ & $E$ & 0,81 & 0,89 & E6 Emotions positives $(0,43)$ & 0,78 & 0,88 \\
\hline & & & & & E1 Chaleur $(0,40)$ & & \\
\hline Activité & 11,16 & $E$ & 0,55 & 0,59 & $\begin{array}{l}\text { E4 Activité }(0,53) \text {; E6 Emotions } \\
\text { positives }(0,53) \text {; E1 Chaleur }(0,51)\end{array}$ & 0,75 & 0,72 \\
\hline Altruisme & $7,22,27 \mathrm{R}, 32$ & $A$ & 0,65 & 0,71 & $\begin{array}{l}\text { A3 Altruisme }(0,64) \text {; A6 Sensibilité } \\
(0,47) \text {; A4 Compliance }(0,47)\end{array}$ & 0,75 & 0,78 \\
\hline Compliance & 2R, 12R, 17 & A & 0,60 & 0,78 & $\begin{array}{l}\text { A4 Compliance }(0,57) ; \text { A3 Altruisme } \\
(0,38) ; \text { A2 Franchise }(0,36)\end{array}$ & 0,72 & 0,85 \\
\hline Ordre & $8 \mathrm{R}, 18 \mathrm{R}$ & C & 0,65 & 0,73 & $\begin{array}{l}\text { C2 Ordre }(0,65) ; \text { C5 Autodiscipline } \\
(0,52) ; \text { C6 Délibération }(0,43)\end{array}$ & 0,68 & 0,72 \\
\hline Autodiscipline & $13,23 R, 28,38,43 R$ & C & 0,73 & 0,78 & $\begin{array}{l}\text { C5 Autodiscipline }(0,71) ; \text { C4 }(0,65) \text {; } \\
\text { C3 }(0,63)\end{array}$ & 0,75 & 0,85 \\
\hline Anxiété & $9 \mathrm{R}, 19,34 \mathrm{R}, 39$ & $\mathrm{~N}$ & 0,86 & 0,87 & $\begin{array}{l}\text { N1 Anxiété }(0,78) \text {; N6 Vulnérabilité au } \\
\text { stress }(0,64) ; \text { N3 Dépression }(0,57)\end{array}$ & 0,84 & 0,88 \\
\hline & & & & & N3 Dépression $(0,55)$; N2 Colère- & & \\
\hline Dépression & 4,29 & $\mathrm{~N}$ & 0,47 & 0,73 & $\begin{array}{l}\text { Hostilité }(0,47) ; \text { N6 Vulnérabilité au } \\
\text { stress }(0,46)\end{array}$ & 0,51 & 0,75 \\
\hline
\end{tabular}




\begin{tabular}{|c|c|c|c|c|c|c|c|}
\hline & & & & & O2 Ouverture à l'esthétique $(0,58)$; & & \\
\hline Esthétique & $30,41 \mathrm{R}, 44$ & $\mathrm{O}$ & 0,65 & 0,79 & $\begin{array}{l}\text { O5 Ouverture aux idées }(0,39) \text {; O1 } \\
\text { Ouverture à l'imagination }(0,32)\end{array}$ & 0,76 & 0,79 \\
\hline Idées & $10,15,25,35 \mathrm{R}, 40$ & $\mathrm{O}$ & 0,69 & 0,76 & $\mathrm{O} 5(0,60) ; \mathrm{O} 1(0,38) ; \mathrm{O} 2(0,38)$ & 0,71 & 0,84 \\
\hline $\begin{array}{l}\text { Facettes spécifiques à } \\
\text { l'échantillon français? }\end{array}$ & & \multicolumn{4}{|c|}{ Facettes spécifiques à } & & \\
\hline Enthousiasme-Fr & $11,16,36$ & $\mathrm{E}$ & 0,62 & 0,82 & $\begin{array}{l}\text { E1 Chaleur }(0.58) \text {; E6 Emotions } \\
\text { positives }(0.58) ; \text { E4 Activité }(0,49)\end{array}$ & 0,76 & 0,78 \\
\hline Compliance-Fr & $2 \mathrm{R}, 12 \mathrm{R}, 17,27 \mathrm{R}$ & A & 0,68 & 0,72 & $\begin{array}{l}\text { A4 Compliance (0.58) ; N2 Colère- } \\
\text { Hostilité }(-0.50) \text {; A3 Altruisme (0.43) }\end{array}$ & 0,64 & 0,86 \\
\hline $\begin{array}{l}\text { Instabilité émotionnelle-Fr } \\
\qquad(24,29)\end{array}$ & 24R, 29 & $\mathrm{~N}$ & 0,72 & 0,65 & $\begin{array}{l}\text { N2 Colère-Hostilité }(0.52) \text {; N3 } \\
\text { Dépression }(0.49) ; \text { N6 Vulnérabilité au } \\
\text { stress }(0.48)\end{array}$ & 0,61 & 0,65 \\
\hline
\end{tabular}

Note. $\mathrm{E}=$ Extraversion $; \mathrm{A}=$ Agréabilité $; \mathrm{C}=$ Conscienciosité ou Caractère consciencieux $; \mathrm{N}=$ Névrosisme $; \mathrm{O}=$ Ouverture 
Figure 1. Résultat du modèle du BFI avec 10 facettes proposé par Soto et John [15] sur les données du BFI-Fr avec la proposition de 3 nouvelles facettes
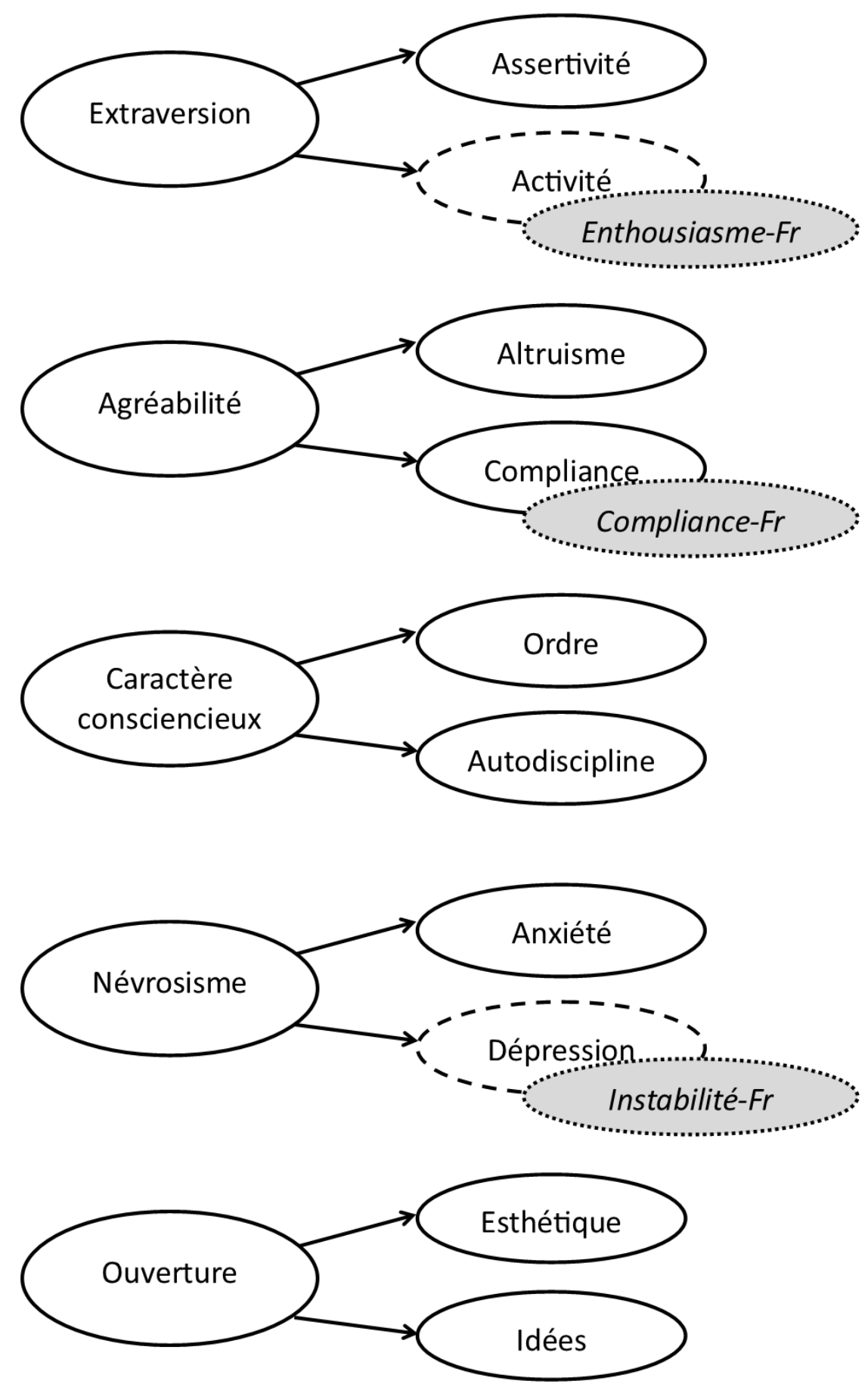

Commentaire : les facettes Activité et Dépression ne présentent pas de consistance interne suffisante. Les facettes Enthousiasme-Fr, Compliance-Fr et Instabilité-Fr représentent des propositions de nouvelles facettes (issues des données relatives au BFI-Fr) avec une meilleure consistance interne. 